\title{
Rotation of solar-like main sequence $\operatorname{stars}^{\star}$
}

\author{
K. Stępień ${ }^{1}$ and E. Geyer ${ }^{2}$ \\ 1 Warsaw University Observatory, Al. Ujazdowskie 4, 00-478 Warszawa, Poland \\ e-mail: kst@astrouw.edu.pl \\ 2 Observatorium Hoher List der Universitäts-Sternwarte, Bonn, D-54550 Daun, Germany
}

Received August 11; accepted October 23, 1995

\begin{abstract}
The results of the photometric survey of 16 solar-type, active, field stars are presented. During our observations 9 stars showed appreciable light variability with amplitudes of a few hundredths of a magnitude but for three of them periods could not be determined. Most of the observed variable stars have periods shorter than about 10 days. It is suggested that, similarly as is observed in the Hyades cluster, small amplitude light variations are quite common among active field solar-type dwarfs with rotation periods around one week or less. A strong modulation of amplitude of some variable stars over the time scale of years is demonstrated. The amplitude may sometimes decrease even below the detectability threshold. A special case is HD 17576 - a visual binary consisting of a G0 dwarf and a much fainter hot subdwarf. It has the largest amplitude of all the stars observed, which suggests an intense spot activity, a very strong $\mathrm{H}$ and $\mathrm{K}$ line core emission and a very high $\mathrm{X}$-ray emission flux, close to the saturation limit. Yet its variability period is equal to 18.74 days - almost an order of magnitude longer than expected for such an active dwarf.
\end{abstract}

Key words: photometry — stars: activity of — stars: rotation of — stars: variable

\section{Introduction}

Since pioneering papers by Wilson (1966) and Kraft (1967) it is generally accepted that single cool stars slow down their rotation during the Main Sequence (MS) life. The spin down is a result of angular momentum loss due to a magnetized stellar wind (Mestel 1984). The following general picture of stellar rotation evolution on the MS seems to be well established (e.g. Stępień 1994). Observations do not show any apparent age dependence of rotation of stars of spectral type earlier than F. The age dependence is increasingly visible over the F type. Single $\mathrm{G}$ and $\mathrm{K}$ stars show a substantial decrease of rotation with age. Scanty observations of M stars are in agreement with a similar decrease of rotation of these stars.

Our Sun, at G2, belongs to stars slowing down noticeably their rotation during the MS life. Its present rotation rate is perfectly normal for its age. Assuming that the Sun is a typical star, its rotation period near the zero age MS should have been close to 2 days (Stȩpień 1991). Is, however, this assumption correct? The present Sun contains only about $0.5 \%$ of the total AM of the Solar System (Allen 1973). Observations of the youngest stellar clusters show that G stars on ZAMS have a broad range of rota-

Send offprint requests to: K. Stȩpień

${ }^{\star}$ Based on observations obtained at ESO, La Silla, Chile tion periods - from a fraction of a day up to a few days but the ultra rapid rotators disappear quickly with time (Soderblom et al. 1993). What was the initial rotation rate for the Sun? Was the Sun an ultra rapid rotator, a moderate rotator or, perhaps, an exceptionally slow rotator with the rotation rate close to the present one? The last possibility seems rather unlikely because all observed young stars have rotation periods shorter than a few days but it cannot be completely excluded because we do not know what is the frequency of occurrence of planetary systems around cool stars and what is the role of the formation of a planetary system on the initial rotation rate of a central star. To understand the rotational history of the Sun more observations of stars similar to it are needed. The present paper deals with a sample of such stars.

\section{Observations}

A group of bright stars with spectral type close to G2 $\mathrm{V}$ was selected for photometric and spectroscopic observations. Here we present and discuss their photometry. A detailed discussion of spectroscopic observations (obtained with the coudé spectrograph of the $1.5 \mathrm{~m}$ ESO telescope) will be published later.

The stars were observed in November 1986 in the $U B V(R I)_{\mathrm{c}}$ system with the $50 \mathrm{~cm}$ ESO telescope and the 
$60 \mathrm{~cm}$ Canadian telescope at the Las Campanas Observatory. The stars were observed in sequences of several stars - one measurement (star-sky-star) at a time and the whole sequence was repeated a few times during a night, with a number of repetitions depending on weather conditions. Including comparison stars for some program stars (see below) did not alter this scheme, except that the comparison stars were observed immediately before, or after the program stars. The observations were transformed to the standard system using standard stars from E-regions (Vogt et al. 1981). The extinction and transformation coefficients were determined for nights with a sufficient number of standards observed (15 on average). For the other nights, with only a fraction of time clear, average coefficients were used. To avoid spending too much time for observations of standards and comparison stars the original program assumed observations of only standard and program stars without comparison stars. In such an all sky survey all the constant stars observed on a given night serve as comparison stars for a given star. If one of program stars turns out to be variable, it is dropped from the list of constant stars and the whole procedure is repeated. Let us describe this procedure in more detail using as an example $V$-magnitude.

After transforming all the measurements from a given night to the standard system, average nightly values for every program star were formed $\langle V\rangle_{i}$, where $i=$ $1,2, \ldots n_{k}$ and $n_{k}$ is the number of the program stars observed on $k$-th night. This eliminates a possibility of detecting variations with time scales shorter than about 2 days. The stars were observed up to 5 times per night (2-3 on average). Then the grand average over all nights, $\overline{V_{j}}$ was found for each star, where $j=1,2, \ldots N$ and $N$ is the total number of the program stars. To search for variability of the $j$-th stars differences between its nightly averages and the nightly averages of all the other stars observed on that particular stars were found and then added to grand averages of the same stars. The final magnitude of the $j$-th star on $k$-th night was determined as the average of the $n_{k}-1$ thus obtained values:

$$
V_{j}^{k}(\text { final })=\frac{\sum_{i=1}^{n_{k}-1}\left(<V>_{j}-<V>_{i}+\overline{V_{i}}\right)}{n_{k}-1}, \quad i \neq j
$$

A fraction of the program stars showed variability (see below). They were removed from the above summation and the whole procedure was repeated until the final magnitudes and color indices were determined.

The above procedure can be treated as an extension of the classical scheme with one or two comparison stars selected exclusively for a given program star. In our case all observed nonvariable stars served as comparison stars for each program star (an exception was HR 88 for which the same comparison star as used by Chugainov 1980 was adopted from the beginning). The scheme saves time and works well under a condition that the observing nights are of a good photometric quality. Summer nights in Chile should fulfil this condition. Unfortunately, 1986 was a year of a poor weather in both observatories; a substantial fraction of the time during our observing run was cloudy. As a result, uninterrupted observations could be obtained only on few whole nights. The accuracy of the adopted procedure drops rapidly with a decreasing number of stars observed on a given night. It was therefore decided after the first week of the run to select comparison stars for several primary target stars for which variability had been reported or could be expected with a high probability. More accurate photometry could be thus secured for these stars. On good nights magnitudes of the program stars calculated from the all sky survey agreed very well with those determined solely from dedicated comparison stars, hence confirming a good efficiency of the assumed scheme. Unfortunately, on poor nights differences were noticeable. In such cases the magnitudes based on individual comparison stars were adopted for those stars for which the comparison stars had been selected. The individual comparison stars used in our program are listed in Table 1. The consecutive columns give their HR and HD numbers, names of the variable stars, the number of nights on which the magnitudes of the respective comparison star were tied up to the standard system, its average magnitudes, mean standard deviation in $V$, and color indices.

In addition to poor weather there appeared instrumental effects on the Canadian telescope, which also influenced the accuracy of measurements. A standard procedure for reduction of photometric observations contains an option for removing possible time drifts of the equipment sensitivity. The drifts observed in ESO were always small or negligible. Unfortunately, they were substantial and in many cases nonlinear in case of the Canadian telescope. Even after removing them, and in spite of the fact that the same set of standard stars was used in both observatories, systematic differences appeared between measurements of a given star obtained with the both telescopes. A closer inspection revealed an existence of the dependence of the instrumental sensitivity on apparent magnitude of a star in a sense that it increased with the brightness. Such a trend could be mimicked e.g. by a substantial negative dead time constant. In our case it was not a result of adopting originally a too big constant - the trend was present even for zero constant. Similar problems with the same photometric equipment were reported by Rucinski (1987). A linear formula in magnitude was fitted and the trend was removed for each night separately.

All these problems lowered substantially the reliability of the Las Campanas observations. In the following analysis they were treated with a lower weight (see below). 
Table 1. Comparison stars

\begin{tabular}{llllllllll}
\hline HR & HD & HD var. & $n$ & $V$ & $\sigma_{V}$ & $B-V$ & $U-B$ & $V-R$ & $V-I$ \\
\hline & 2488 & 1835 & 15 & 6.840 & 0.011 & 0.532 & 0.054 & 0.308 & 0.607 \\
863 & 18149 & 17576 & 9 & 5.922 & 0.009 & 0.442 & 0.038 & 0.259 & 0.503 \\
1007 & 20791 & 20630 & 9 & 5.673 & 0.010 & 0.962 & 0.757 & 0.490 & 0.925 \\
2220 & 43042 & 39587 & 13 & 5.192 & 0.009 & 0.443 & 0.001 & 0.265 & 0.520 \\
2290 & 44594 & 41824 & 9 & 6.623 & 0.023 & 0.647 & 0.215 & 0.354 & 0.680 \\
\hline
\end{tabular}

\section{Analysis}

\subsection{Method of analysis}

The observed stars were checked for variability by comparing the mean standard deviation of each star $V$ measurements with the mean standard error of observations of constant stars. The latter has a value of 0.008 of a magnitude. This value was calculated from observations obtained in the both observatories, taken together. Residual systematic shifts, still present between both sets of observations for some stars (see below) increased the scatter and influenced the value of the standard error. Errors of color indices are $0.006,0.010,0.006$ and 0.013 of a magnitude for $B-V, U-B, V-R$ and $V-I$, respectively.

Stars with mean standard deviations less than 1.5 standard errors, i.e. 0.011 of a magnitude, were treated as not showing variability. All the other stars were assumed to show variability and a period analysis was performed for them. Because the LC observations were of inferior quality and sometimes systematically shifted relative to the ESO observations, which, of course, increases uncertainty, an additional check for reality of periodic variations was applied: the ESO observations alone had to show the same periodicity.

The period analysis was restricted to periods longer than 2 days and shorter than 20 days. The upper limit excluded a possible detection of a spurious periodicity resulting from a shift between the ESO and LC observations whereas the lower limit resulted from sampling of data.

Summary of the analysis is given in Table 2. The consecutive columns give HR and HD numbers of a star, its spectral type, the number of nights on which the star was observed, the average values of $V$-magnitude, their standard deviations, average color indices and the conclusion of the variability analysis. Figure 1 shows the light curves of the periodic variables. Only $V$-curves are shown because color indices show nothing but a scatter. A detailed discussion of each star is given below. It should be stressed that classification of some of the stars as variable should still be treated as tentative even when a variation period could be determined: in many cases the resulting amplitudes of the light curves were equal to about 0.015 of a magnitude, i.e. only two times more than the standard error. With a number of observations hardly exceeding 10 the probability of detecting a spurious periodicity is rather high. Individual observations are listed in Table 3.

\subsection{Discussion of individual stars}

HR $88=$ BE Cet. This is a well known active star. Chugainov (1980) discovered its light variations with a period of 7.655 days. He interpreted these variations as a result of rotational modulation of spotted surface of the star. During his observations in 1977-78 the amplitude of variation was equal to 0.032 and $0.037 \mathrm{mag}$ in $V$ and $B$ filters, respectively. Noyes et al. (1984) observed periodic calcium emission variation with a period of 7.7 days. HR 88 is one of few stars of our sample for which measurements of the surface magnetic field have been obtained. The resulting surface averaged magnetic field $\langle f B\rangle=$ 450 G (Saar 1990). This was also the only our star for which an individual comparison star, HD 2488 (Table 1), had been selected and observed from the beginning of our run. Our observations of the variable show a surprisingly small scatter, of the same order as the mean observational error. Had we not known the star to be variable in brightness, we would have classified it as nonvariable. The $V$ observations are plotted in Fig. 1 with the Chugainov period of 7.655 days. Both the amplitudes, in $V$ and $B$, are close to 0.015 of a magnitude, but the $B$ amplitude may be slightly larger, by $0.002-0.003$ of a magnitude. In late eighties the star was observed photometrically by Cutispoto $(1991,1992,1995)$. The amplitude varied from season to season (see below). Our blue spectra confirm an existence of a strong $\mathrm{H}$ and $\mathrm{K}$ line core emission with a variable profile.

HR 159. The star has a low level of chromospheric activity (Pasquini 1992). The value of the expected rotation period, corresponding to such a low activity, is 32.6 days (Noyes et al. 1984). The scatter of our observations is 0.005 of a magnitude, which is less than the average error. The star does not show any light variability. A weak emission profiles are visible in $\mathrm{H}$ and $\mathrm{K}$ lines with a complex structure.

HR 173. In many respects the star is very similar to HR 159. The calculated value of the rotation period is 31.8 days (Noyes et al. 1984). Our observations show a small scatter of 0.007 of a magnitude, hence we conclude that 
Table 2. Program stars

\begin{tabular}{|c|c|c|c|c|c|c|c|c|c|c|}
\hline HR & HD & $\mathrm{Sp}$ & $n$ & $<V>$ & $\sigma_{V}$ & $<B-V>$ & $<U-B>$ & $<V-R>$ & $<V-I>$ & variability \\
\hline 88 & 1835 & $\mathrm{G} 2 \mathrm{~V}$ & 15 & 6.370 & 0.007 & 0.664 & 0.220 & 0.371 & 0.710 & $P=7.655$ (assumed) \\
\hline 159 & 3443 & G8V & 14 & 5.565 & 0.005 & 0.717 & 0.251 & 0.402 & 0.775 & nonvar. \\
\hline \multirow[t]{2}{*}{173} & 3795 & G3V & 14 & 6.127 & 0.007 & 0.720 & 0.186 & 0.403 & 0.794 & nonvar. \\
\hline & 11131 & dG1 & 15 & 6.716 & 0.008 & 0.632 & 0.112 & 0.356 & 0.703 & nonvar. \\
\hline \multirow[t]{2}{*}{695} & 14802 & G1V & 14 & 5.181 & 0.011 & 0.615 & 0.119 & 0.350 & 0.687 & nonvar? \\
\hline & 17576 & G0V & 16 & 7.831 & & 0.577 & -0.148 & 0.364 & 0.737 & $P=18.75$ \\
\hline 996 & 20630 & G5V & 12 & 4.838 & 0.011 & 0.677 & 0.202 & 0.380 & 0.731 & nonvar. \\
\hline 1321 & 26913 & G5V & 13 & 6.920 & 0.011 & 0.689 & 0.217 & 0.381 & 0.737 & $P=6.7$ \\
\hline 1322 & 26923 & G0V & 13 & 6.299 & 0.007 & 0.579 & 0.059 & 0.329 & 0.643 & nonvar. \\
\hline 1532 & 30495 & G1V & 12 & 5.474 & 0.005 & 0.637 & 0.140 & 0.359 & 0.690 & nonvar. \\
\hline 1780 & 35296 & $\mathrm{~F} 8 \mathrm{~V}$ & 12 & 5.002 & 0.011 & 0.533 & 0.006 & 0.311 & 0.604 & $P \sim 4^{\mathrm{d}}$ \\
\hline 2047 & 39587 & G0V & 13 & 4.398 & 0.008 & 0.585 & 0.071 & 0.341 & 0.671 & $P=5.5$ \\
\hline 2162 & 41824 & G6V & 12 & 6.646 & 0.014 & 0.720 & 0.277 & 0.410 & 0.787 & var. \\
\hline 2208 & 42807 & $\mathrm{G} 2 \mathrm{~V}$ & 12 & 6.456 & 0.015 & 0.675 & 0.186 & 0.369 & 0.716 & $P=7^{\mathrm{d}}-8^{\mathrm{d}}$ \\
\hline 3064 & 64096 & G0V & 10 & 5.156 & 0.011 & 0.605 & 0.083 & 0.346 & 0.668 & $P=10^{\mathrm{d}} ?, 22^{\mathrm{d}} ?$ \\
\hline 3538 & 76151 & G3V & 9 & 6.014 & 0.013 & 0.664 & 0.222 & 0.367 & 0.698 & var.? \\
\hline
\end{tabular}

the star is nonvariable. $\mathrm{H}$ and $\mathrm{K}$ line profiles similar to HR 159.

HD 11131. The star is relatively active: with $\log F_{\mathrm{MgII}}$ $=6.56$ (Rutten et al. 1991) the expected rotation period is close to 10 days (Stępień 1994). Yet our observations have a small scatter of 0.008 of a magnitude, which indicates a lack of variability. Medium strong $\mathrm{H}$ and $\mathrm{K}$ line emission is visible in our spectra with the K-line emission red-shifted.

HR 695. According to Rutten et al. (1991) the star is quite active: its net calcium emission flux is equal to 6.46dex which corresponds to a rotation period about 10 days (Stępien 1989). This is in a fair agreement with the measurements of Soderblom (1985), according to whom the calculated rotation period $P_{\text {calc }} \approx 9$ days. However, the value of the net K-line flux determined by Pasquini (1992) is equal to only $5.55 \mathrm{dex}$, which gives approximately 5.85 dex for the emission flux in both calcium lines. Such a low value would suggest a rotation period longer than 20 days. The $v \sin i=4 \mathrm{~km} / \mathrm{s}$ (Hoffleit \& Jaschek 1982) favors a faster rotation. Our observations showed a noticeable systematic difference between the ESO and LC observations: $V=5.188$ and 5.172 for the average magnitudes measured in ESO and LC, respectively. The color indices did not show any systematic difference. The internal scatter within each series was, however, small. This leads to a conclusion that either the star was nonvariable, and the shift was of instrumental origin, or the star was variable on a scale of one month. Cautiously, we adopt the first possibility as a more probable explanation. No $\mathrm{H}$ and $\mathrm{K}$ line emission is visible in our spectra.

HD 17576. The star was included into our program at the suggestion of Dr. E.H. Olsen who had demonstrated its variability (1980) but, due to an insuffucient number of observations, was unable to determine the variation period. After the first week of observations we added an individual comparison star, HR 863 (Table 1), to the variable. HD 17576 belongs to very active stars: its $\log F_{x} \approx 7 \mathrm{erg} / \mathrm{cm}^{2} \mathrm{~s}$ is at the saturation level (Hempelmann et al. 1995). It is a visual binary consisting of G0 dwarf (or marginal subgiant) and a hot subdwarf lying nearly 2 arcsec from the primary. The subdwarf is about 3 magnitudes fainter in the $V$ band but because of a high temperature it contributes significantly to the $U$ band producing a peculiar $U-B$ (Table 2 ). The star is definitely variable. Our observations alone suggest a period around 20 days. They can be folded together with the Olsen (1980) y observations of the uvby system with a period of 18.75 days. Figure 1 shows the resulting light curve. The $V$ amplitude is 0.06 of a magnitude. The $B$ amplitude is similar but defined much poorer, from our observations only. The $\mathrm{H}$ and $\mathrm{K}$ line emission is strong. Details of the profiles could not be seen due to a poor focusing of the plate.

HR $996=\kappa^{1}$ Cet. The star is chromospherically and coronally active (Rutten et al. 1991). According to Noyes et al. (1984) its rotation period is equal to 9.4 days. From the observations over a longer time interval Donahue (1993) obtained the improved value of 9.2 days. The surface averaged magnetic field has an intensity of 525 G (Saar 1990). Our observations have a scatter of nearly 0.012 of a magnitude, close to our limit of variability. We classify the star as not showing any significant light variations. A blue spectrum shows a strong $\mathrm{H}$ and $\mathrm{K}$ line emission.

HR 1321 = V891 Tau. The star was observed photometrically by Blanco et al. (1979) in early seventies. Analysing the $U B V$ observations obtained on several nights the authors did not see any excessive scatter and concluded that the star was constant. However, the observations obtained early in 1984 showed the star to be variable with a period of 6.8 days (Ziegler et al. 1984). 

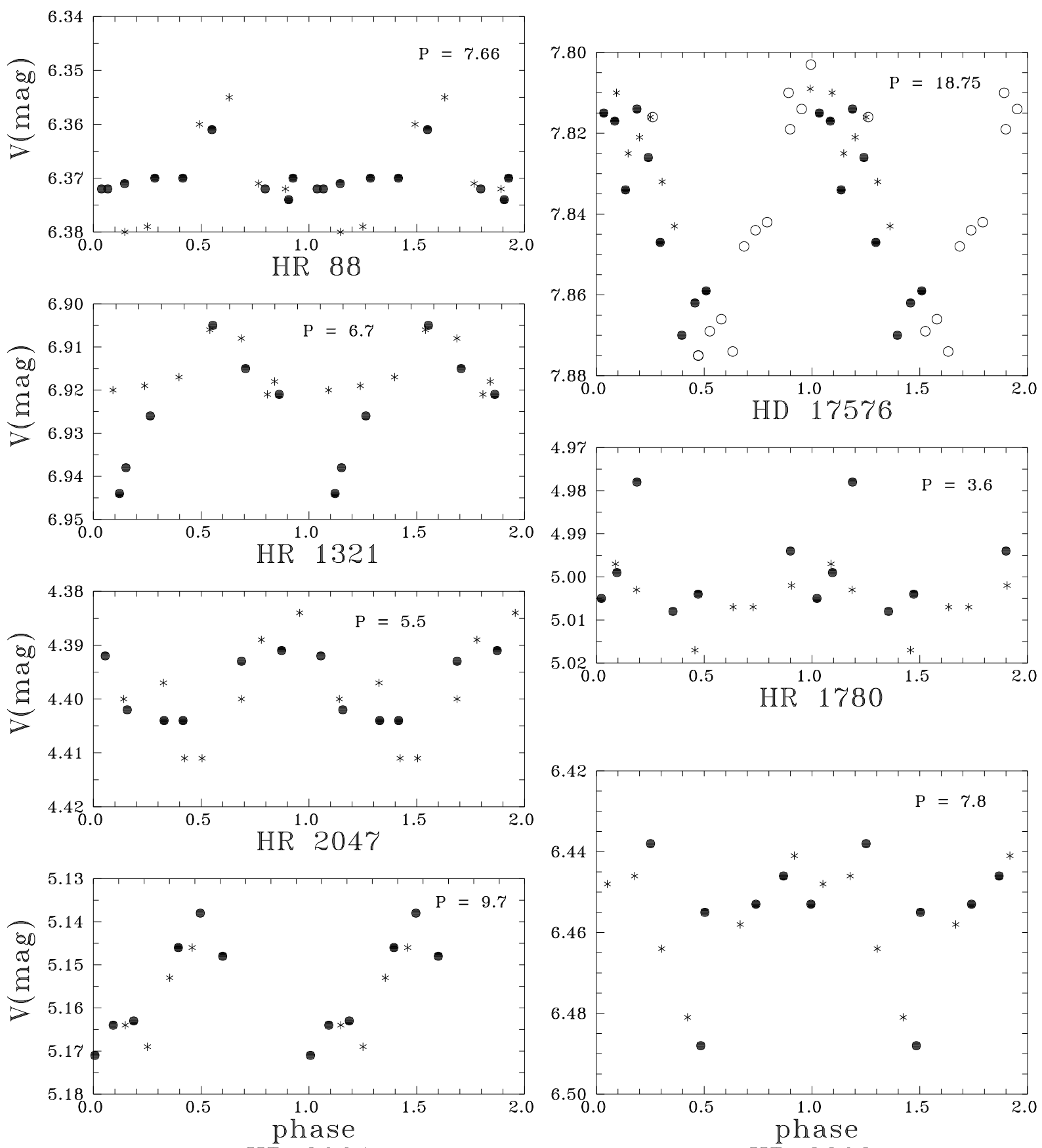

HR 3064

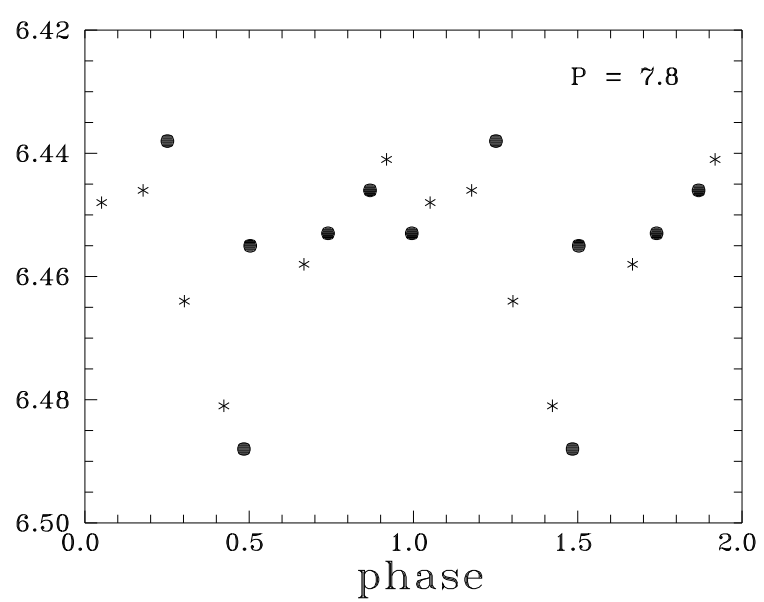

HR 2208

Fig. 1. $V$ magnitudes of periodic variables plotted with arbitrary zero points and indicated periods: filled circles denote ESO observations, asterisks - LC observations and open circles are the $y$ observations of HD 17576 obtained by Olsen (1980)

Noyes et al. (1984) gives the rotation period of this star determined from calcium emission modulation, $P_{\text {rot }}=7.2$ days. Our observations suggest a small amplitude variations with a period of 6.7 days, in a good agreement with the results by Ziegler et al. (1984). The $V$ observations are plotted in Fig. 1. The scatter around the light curve is considerable, hence the amplitude is poorly determined. Its value is 0.02 of a magnitude - two and a half times less than obtained by Ziegler et al. (1984). The star was later observed by Cutispoto $(1991,1995)$ and showed an even lower amplitude of light variations. A medium strong emission in $\mathrm{H}$ and $\mathrm{K}$ lines is visible in our spectrum.

HR 1322 = V744 Tau. The star forms a visual binary system with HR 1321. The separation of the components is about 1 arcmin. Its rotation period, expected from the average calcium emission flux, is $P_{\text {calc }}=5.6$ days (Noyes et 
al. 1984). Blanco et al. (1979) noted a substantially larger scatter of photometric observations of HR 1322 compared to HR 1321. They concluded that the star was variable. Our observations show the contrary. The scatter of the observations of HR 1322 was only 0.007 of a magnitude which is less than the average observational error. We conclude that the star did not show any measurable variability during our observing run. The spectrum shows a slight $\mathrm{H}$ and $\mathrm{K}$ line emission.

HR $1532=58$ Eri. The star is a well known active dwarf. Its rotation period $P_{\text {rot }}=7.6$ days was determined by Noyes et al. (1984). The scatter of our observations was the smallest of all the observed program stars - only 0.005 of a magnitude. This indicates that the star was nonvariable. A marginal $\mathrm{H}$ and $\mathrm{K}$ line emission could be seen in our spectrum.

HR $\mathbf{1 7 8 0}=111$ Tau. This is a relatively hot dwarf with a high activity level (Herbig 1985; Rutten et al. 1991; Hempelmann et al. 1995). The star has also been detected in extreme UV (Pounds et al. 1993). Donahue (1993) found its rotation period $P_{\text {rot }}=3.6$ days from a modulation of calcium emission flux. Our observations show a scatter larger than the defined by us limit of nonvariability. Periodogram shows a broad maximum around 4-5 days. The data are plotted in Fig. 1 with the Donahue period of 3.6 days. This should not be treated as an independent confirmation of this period; the light curve is poorly defined and looks in fact slightly better with a period of 4.3 days. The amplitude is about 0.015 of a magnitude which places the star among marginal variables. The spectrum shows a strong $\mathrm{H}$ and $\mathrm{K}$ line emission with broad and complex profiles.

HR $2047=\chi^{1}$ Ori. This is probably the most often observed star among our program stars. Its high brightness $(V=4.4)$ and an easy access from observatories in both hemispheres make the star a favorable target of many observational programs. Its rotation period $P_{\text {rot }}=5.2$ days (Noyes et al. 1994) is in a good agreement with $v \sin i=$ $9.4 \mathrm{~km} / \mathrm{s}$ (Simon \& Drake 1989). Its surface averaged magnetic fields has a value of $600 \mathrm{G}$ (Saar 1990). HR 2047 was the most northern star in our program and was observed through systematically higher air masses than the other stars. After the first week of observations an individual comparison star, HR 2220 (Table 1), was selected for the variable. The scatter exceeded significantly the observational error, which was an indication of variability. However, in addition to an excessive scatter, a substantial systematic shift appeared between the ESO and LC observations. A closer inspection of individual observations of both, the variable and the comparison star revealed that the variable was systematically brighter during the LC run, whereas the comparison star was fainter. The resulting LC var-comp magnitudes differed on average by 0.020 of a magnitude from the ESO observations. They were arbitrarily corrected by this amount and the thus obtained magnitudes of the variable analysed, although the "corrected" scatter is now less than 0.011 of a magnitude. Period analysis showed a maximum at the value of 5.5 days, in a good agreement with the value determined from calcium emission modulation. The amplitude of the $V$-curve has a marginal value of 0.015 of a magnitude (Fig. 1 ). The conclusion about the presence of the periodic photometric variability of this star should be treated with a particular caution and it needs an independent confirmation. Our spectroscopic observations confirm the presence of a prominent $\mathrm{H}$ and $\mathrm{K}$ line emission with complex and broad profiles.

HR 2162. The star was included into our program as one of the primary target stars because of a remark in the Bright Star Catalogue that it may be variable with an amplitude of 0.1 of a magnitude (Hoffleit \& Jaschek 1982). It is a tight visual binary and Pounds et al. (1993) remark that the B component may be an RS CVn type star. After a few days an individual comparison star, HD 2290 (Table 1), was selected for the variable. An absolute photometry showed that HR 2162 was fainter by about 0.02 of a magnitude during the LC observations but HD 2290 was at the same time fainter by almost 0.04 of a magnitude! The resulting magnitudes of the variable, calculated from the var-comp differences, showed the variable to be brighter when observed in the LC. We were unable to decide about variability of either of the observed stars. The internal scatter of the ESO or LC observations taken separately was in both cases of the order of the observational errors which rules out the 0.1 magnitude variability of $\mathrm{HR}$ 2162 on a time scale shorter than a few weeks. The star has a strong $\mathrm{H}$ and $\mathrm{K}$ line emission.

HR 2208. This star attracted so far little attention of observers. It was included into our program because of a high activity level. The average calcium emission flux of this star corresponds to a rotation period of about 8 days (Soderblom 1985). Our observations showed a noticeable scatter, resulting very likely from the photometric variability of the star. Search for periodicity gave a maximum probability around 7-8 days. The data are plotted in Fig. 1 with a period of 7.8 days. The amplitude of variation is about 0.03 of a magnitude in $V$ and 0.035 of a magnitude in $B$. HR 2208 has thus the second largest amplitude of light variation detected in the present survey. The blue spectrum of this star showed a strong $\mathrm{H}$ and $\mathrm{K}$ line emission with broad, complex profiles.

HR 3064 = 9 Pup. The star has a low activity level. Its rotation period, estimated from the calcium emission flux (Pasquini 1992), should be around 20 days. Our observations show an unexpectedly substantial scatter, suggestive of variability. The best period, within the investigated range, is about 10 days. Figure 1 presents the data plotted with the period of 9.7 days. The $V$ light curve has an amplitude of 0.02 of a magnitude. A visual inspection of the observations shows that the data can also be folded 
with a period of about 22 days. That value is outside of the investigated range (2-20 days), see Sect. 3.1. In the case of HR 3064 no systematic shift between the ESO and $\mathrm{LC}$ observations is present. The star deserves further observations. Only K-line shows a marginal emission on our spectrum which was slightly out of focus.

HR 3538. The expected value of the rotation period of this star is 15 days (Noyes et al. 1984). It has a moderate X-ray flux, compatible with the calculated period (Rutten et al. 1991). Our observations show a scatter significantly exceeding the observational errors but the star was observed on only 9 nights. No period analysis was therefore performed but a visual inspection of the observations suggests a value of a period in the vicinity of 10 days. More observations would be necessary to settle down the value of the variability period.

\section{Discussion}

The results of the present survey demonstrate that periodic light variations are not exceptional among active, solar type field dwarfs. There were 6 stars in our program with known rotation periods shorter than 10 days. Four stars among them showed appreciable periodic light variations during our observations. Their periods and amplitudes are similar to those of solar type members of the Hyades cluster (Radick et al. 1987). In addition to those 6 stars with measured periods, the calcium emission flux level of 3 more stars indicates the rotation period shorter than 10 days. These are HD 11131, HR 695 and HR 1322. None of them showed, however, any measurable light variations during our observing run. We also observed two stars with long known rotation periods around 32 days. Both were constant. The measured calcium emission flux of HR 3064 and HR 3538 suggests a rotation period around 15-20 days. We could see an indication of a possible variability in both cases but no unique period could be determined.

An interesting result of our survey is the demonstration of long term variability of an amplitude of light variations of some active stars. Such a variability, often associated with a variability of an average brightness, has been observed for a number of heavily spotted, rapidly rotating stars of BY Dra or RS CVn type (Bohusz \& Udalski 1981; Pettersen et al. 1991; Cutispoto 1995 and references therein). It is usually assumed that both result from a cyclic nature of activity. Less is known about long term variability of solar type field dwarfs. Radick et al. (1983) noted seasonal variability of amplitudes of several members of the Hyades cluster. The brightness of some of active stars monitored at the Lowell Observatory shows slow variations of the order of a fraction of percent, which are correlated with the chromospheric activity index (Zhang et al. 1994). Our data demonstrate that an amplitude of the rotational modulation of stellar brightness of moderately active G- type field stars also varies on a long time scale. Two most convincing examples are HR 88 and HD 1321.

The amplitude of light variations of HR 88 decreased from 0.032 down to 0.015 of a magnitude, i.e. by a factor of two, between 1977-78 and 1986. In 1987-90 the amplitude increased again and varied between 0.03 and 0.04 of a magnitude (Cutispoto 1991, 1992, 1995). The star has a fairly well defined activity cycle of the length of 9.1 year, which is superimposed on a systematic decrease of activity present since at least mid- sixties (Baliunas et al. 1995). Chugainov (1980) had obtained his observations of HR 88 almost exactly one cycle earlier than we did. This means that the both sets of observations pertain to the same phase of the activity cycle, close to a mid-time between activity maxima (Baliunas et al. 1995, Fig. 1d).

HR 1321 has a similar behavior of the $S$-index to HR 88. A cycle of length of 7.8 year is fairly visible in the data, together with a systematic decrease of activity (Baliunas et al. 1995). Our observations give an amplitude of light variation about two and a half times smaller than is visible in the data obtained by Ziegler et al. (1984) in 1984, i.e. only two years earlier. In 1987 and 1989 the amplitude was even smaller and did not exceed 0.015 of a magnitude (Cutispoto 1991, 1995). The visual inspection of Fig. 1e in Baliunas et al. (1995) does not show any apparent correlation between the amplitude of light variations and a phase of activity cycle. HR 1321 had also been observed by Blanco et al. (1979) during the interval 1970-74. They could not detect any light variations exceeding the measurement error but the plot of individual measurements shows a scatter of a few hundredths of a magnitude. Unfortunately, the authors did not publish numerical data. In early seventies the $S$-index was higher by about 0.04 compared to 1984-86. Clearly, the relation between the instantaneous amplitude of light variations and the mean calcium emission level averaged over several rotation periods is not straightforward.

HD 17576 deserves a special attention. Darius \& Whitelock (1978) classified the primary of this visual binary as G0 V or G0 V-IV. If the very high activity and the observed light variations of HD 17576 are due to the primary star, its MS position cannot be reconciled with a rotation period of nearly 19 days. Dwarfs showing the chromospheric-coronal activity at the saturation level and substantial light variations rotate typically with periods of a few days, i.e. nearly an order of magnitude less than the derived period of HD 17576. High activity, together with longer rotation period, is characteristic of evolved stars. To check the evolutionary status of HD 17576 additional observations are necessary.

\section{Conclusions}

The results of the photometric survey of 16 solar type field dwarfs are presented and discussed. Light variability was shown by 9 stars during our observing run. Nearly 
Table 3. Photometry of the program stars. Observations obtained on JD $<2446750$ are from ESO and the others are from LC

\begin{tabular}{|c|c|c|c|c|c|c|c|c|c|c|c|}
\hline $\begin{array}{l}\text { JD } \\
2446700+ \\
\end{array}$ & $\begin{array}{l}V \\
\mathrm{mag} \\
\end{array}$ & $\begin{array}{l}B-V \\
\text { mag }\end{array}$ & $\begin{array}{l}U-B \\
\text { mag }\end{array}$ & $\begin{array}{l}V-R \\
\text { mag }\end{array}$ & $\begin{array}{l}V-I \\
\text { mag } \\
\end{array}$ & $\begin{array}{l}\text { JD } \\
2446700+ \\
\end{array}$ & $\begin{array}{l}V \\
\text { mag }\end{array}$ & $\begin{array}{l}B-V \\
\text { mag }\end{array}$ & $\begin{array}{l}U-B \\
\mathrm{mag}\end{array}$ & $\begin{array}{l}V-R \\
\text { mag } \\
\end{array}$ & $\begin{array}{l}V-I \\
\text { mag }\end{array}$ \\
\hline & & & HR 88 & & & 54.817 & 4.997 & .532 & .002 & .304 & .593 \\
\hline 36.724 & 6.374 & .671 & .217 & .377 & .712 & 56.781 & 5.007 & .541 & .014 & .309 & .609 \\
\hline 37.734 & 6.372 & .658 & .229 & .377 & .710 & 57.758 & 5.002 & .530 & -.001 & .307 & .600 \\
\hline 38.557 & 6.371 & .661 & .218 & .373 & .712 & 58.769 & 5.003 & .551 & -.006 & .298 & .592 \\
\hline 39.628 & 6.370 & .668 & .229 & .368 & .707 & 59.746 & 5.017 & .529 & .008 & .313 & .612 \\
\hline 40.625 & 6.370 & .671 & .217 & .370 & .707 & 60.719 & 5.007 & .532 & .009 & .313 & .611 \\
\hline 41.658 & 6.361 & .665 & .217 & .361 & .703 & & HR 2047 & & & & \\
\hline 43.549 & 6.372 & .673 & .218 & .371 & .711 & 36.863 & 4.402 & .581 & .062 & .340 & .663 \\
\hline 44.530 & 6.370 & .671 & .223 & .372 & .713 & 37.804 & 4.404 & .588 & .047 & .342 & .669 \\
\hline 45.613 & 6.372 & .662 & .221 & .369 & .712 & 39.777 & 4.393 & .594 & .074 & .344 & .670 \\
\hline 54.669 & 6.379 & .658 & .222 & .378 & .718 & 40.800 & 4.391 & .593 & .068 & .337 & .658 \\
\hline 56.523 & 6.360 & .657 & .223 & .369 & .704 & 41.801 & 4.392 & .575 & .091 & .365 & .676 \\
\hline 57.578 & 6.355 & .663 & .208 & .369 & .711 & 43.787 & 4.404 & .585 & .075 & .343 & .674 \\
\hline 58.619 & 6.371 & .665 & .216 & .372 & .706 & 54.825 & 4.411 & .578 & .057 & .343 & .692 \\
\hline 59.572 & 6.372 & .663 & .220 & .369 & .711 & 56.787 & 4.389 & .588 & .069 & .340 & .679 \\
\hline \multirow[t]{2}{*}{61.525} & 6.380 & .659 & .222 & .371 & .711 & 57.766 & 4.384 & .583 & .067 & .339 & .673 \\
\hline & & & HD 17576 & & & 58.778 & 4.400 & .577 & .077 & .342 & .666 \\
\hline 36.802 & 7.815 & .575 & -.144 & .361 & .737 & 59.787 & 4.397 & .588 & .059 & .334 & .669 \\
\hline 37.758 & 7.817 & .580 & -.144 & .357 & .746 & 60.773 & 4.411 & .586 & .083 & .337 & .674 \\
\hline 38.710 & 7.834 & .579 & -.140 & .361 & .732 & 61.776 & 4.400 & .593 & .099 & .328 & .665 \\
\hline 39.688 & 7.814 & .578 & -.131 & .362 & .741 & & HR 2208 & & & & \\
\hline 40.682 & 7.826 & .575 & -.141 & .363 & .739 & 37.812 & 6.488 & .682 & .187 & .310 & .696 \\
\hline 41.718 & 7.847 & .558 & -.111 & .378 & .766 & 39.813 & 6.453 & .683 & .199 & .373 & .711 \\
\hline 43.602 & 7.870 & .572 & -.144 & .367 & .757 & 40.810 & 6.446 & .681 & .200 & .374 & .707 \\
\hline 44.742 & 7.862 & .573 & -.161 & .359 & .744 & 41.801 & 6.453 & .667 & .215 & .385 & .698 \\
\hline 45.714 & 7.859 & .579 & -.134 & .364 & .737 & 43.800 & 6.438 & .670 & .189 & .376 & .712 \\
\hline 54.771 & 7.809 & .573 & -.175 & .358 & .730 & 45.761 & 6.455 & .679 & .192 & .375 & .710 \\
\hline 56.671 & 7.810 & .595 & -.169 & .368 & .734 & 54.844 & 6.458 & .671 & .183 & .370 & .717 \\
\hline 57.679 & 7.825 & .576 & -.145 & .365 & .727 & 56.802 & 6.441 & .677 & .176 & .374 & .727 \\
\hline 58.664 & 7.821 & .578 & -.141 & .368 & .724 & 57.839 & 6.448 & .665 & .167 & .372 & .727 \\
\hline 59.635 & 7.816 & .582 & -.155 & .368 & .733 & 58.823 & 6.446 & .671 & .170 & .361 & .720 \\
\hline 60.635 & 7.832 & .586 & -.169 & .368 & .730 & 59.800 & 6.464 & .674 & .179 & .376 & .736 \\
\hline \multirow[t]{2}{*}{61.711} & 7.843 & .572 & -.158 & .358 & .720 & 60.739 & 6.481 & .679 & .177 & .377 & .735 \\
\hline & & & HR 1321 & & & & & HR 3064 & & & \\
\hline 36.810 & 6.944 & .698 & .212 & .380 & .740 & 36.893 & 5.164 & .603 & .085 & .339 & .665 \\
\hline 37.766 & 6.926 & .693 & .215 & .378 & .732 & 37.816 & 5.163 & .610 & .070 & .341 & .651 \\
\hline 39.709 & 6.905 & .688 & .229 & .382 & .737 & 39.817 & 5.146 & .608 & .089 & .344 & .664 \\
\hline 40.729 & 6.915 & .692 & .230 & .385 & .717 & 40.814 & 5.138 & .608 & .084 & .342 & .658 \\
\hline 41.774 & 6.921 & .674 & .235 & .396 & .754 & 41.821 & 5.148 & .594 & .111 & .364 & .692 \\
\hline 43.710 & 6.938 & .693 & .220 & .388 & .742 & 45.769 & 5.171 & .605 & .087 & .348 & .668 \\
\hline 54.807 & 6.921 & .686 & .204 & .377 & .735 & 56.839 & 5.164 & .604 & .086 & .346 & .669 \\
\hline 56.708 & 6.920 & .691 & .211 & .382 & .741 & 57.833 & 5.169 & .604 & .076 & .345 & .671 \\
\hline 57.696 & 6.919 & .691 & .212 & .383 & .743 & 58.831 & 5.153 & .605 & .069 & .342 & .673 \\
\hline 58.757 & 6.917 & .691 & .205 & .370 & .733 & 59.839 & 5.146 & .607 & .077 & .349 & .671 \\
\hline 59.717 & 6.906 & .680 & .210 & .377 & .736 & & HR 3538 & & & & \\
\hline 60.694 & 6.908 & .688 & .217 & .381 & .746 & 36.899 & 6.015 & .668 & .226 & .366 & .684 \\
\hline \multirow[t]{2}{*}{61.738} & 6.918 & .688 & .218 & .370 & .731 & 37.819 & 6.028 & .661 & .208 & .373 & .712 \\
\hline & & & HR 1780 & & & 39.844 & 5.996 & .676 & .234 & .362 & .679 \\
\hline 36.842 & 4.999 & .530 & .002 & .308 & .594 & 40.822 & 6.004 & .661 & .219 & .370 & .691 \\
\hline 37.777 & 5.008 & .534 & -.004 & .311 & .611 & 41.824 & 5.997 & .655 & .257 & .382 & .715 \\
\hline 39.741 & 4.994 & .541 & .001 & .307 & .602 & 56.847 & 6.027 & .672 & .220 & .361 & .692 \\
\hline 40.778 & 4.978 & .530 & .009 & .306 & .590 & 57.847 & 6.030 & .660 & .213 & .362 & .704 \\
\hline 41.798 & 5.004 & .517 & .039 & .340 & .637 & 58.838 & 6.015 & .658 & .202 & .361 & .703 \\
\hline 43.781 & 5.005 & .530 & .003 & .314 & .603 & 59.845 & 6.012 & .661 & .222 & .368 & .704 \\
\hline
\end{tabular}


all of them have periods shorter than 10 days. We conclude that a substantial fraction of solar type dwarfs with periods of several days shows light variations with amplitudes of the order of 1-2 percent. A few of our stars had been observed earlier. Comparison of our photometry with the older results reveals a presence of substantial, long term variability of the amplitude of a light curve. A ubiquitous presence of limited light variations with a highly variable amplitude among active solar type field dwarfs demonstrates a close similarity of these stars to members of young clusters (Radick et al. 1983, 1987).

One of the program stars, HD 17576, turned out to be exceptional. It showed the largest amplitude of all the investigated stars, yet its variation period is rather long nearly 19 days. The star has an extremely high level of Xray emission (Hempelmann et al. 1995) and strong $\mathrm{H}$ and $\mathrm{K}$ line emission. If its observed properties are interpreted in a standard way as resulting from activity of a primary component of visual binary, rotating with a period of 19 days, its present spectral classification (G0 V or G0 IV$\mathrm{V})$ results in a violation of the period-activity relation obeyed by dwarfs (e.g. Stȩpień 1994). Additional, accurate observations of this star are needed to resolve the present contradiction.

Acknowledgements. We thank Dr. E.H. Olsen for calling our attention to HD 17576, Ms. Ewa Bohusz for a substantial help in reducing the observations and Dr. K. Jahn for help in period analysis. Special thanks are due Dr. G. Cutispoto, the referee, for his discerning opinion about the earlier version of the paper. This research was partly supported by a grant KBN 2 P304 004 007.

\section{References}

Allen C.W., 1973, Astrophysical Quantities, 3rd ed. The Athlone Press, London

Baliunas S.L., et al., 1995, ApJ 438, 269

Blanco C., Catalano S., Marilli E., 1979, A\&AS 36, 297

Bohusz E., Udalski A., 1981, Acta Astron. 31, 185

Chugainov P.F., 1980, Izv. Crim. Obs. 61, 124

Cutispoto G., 1991, A\&AS 89, 435

Cutispoto G., 1992, A\&AS 95, 397

Cutispoto G., 1995, A\&AS 111, 507
Darius J., Whitelock P.A., 1978, Nat 275, 428

Donahue R.A., 1993, PhD Thesis, New Mexico State University

Hempelmann A., Schmitt J.H.M.M., Schultz M., Rüdiger G., Stȩpień K., 1995, A\&A 294, 515

Herbig G.H., 1985, ApJ 289, 269

Hoffleit D., Jaschek C., 1982, The Bright Star Catalogue, IVth ed., Yale University Observatory, New Haven

Kraft R.P., 1967, ApJ 150, 551

Mestel L., 1984, Cool Stars, Stellar Systems, and the Sun . In: Baliunas S.L., Hartmann L. (eds.). Springer, Berlin, p. 49

Noyes R.W., Hartmann L.W., Baliunas S.L., Duncan D.K., Vaughan A.H., 1984, ApJ 279, 763

Olsen E.H., 1980, IBVS No. 1770

Pasquini L., 1992, A\&A 266, 347

Pettersen B.R., Olah K., Sandmann W.H., 1991, in The Sun and Cool Stars: activity, magnetism, dynamos. In: Tuominen I., Moss D., Rüdiger G. (eds.). Springer, Berlin, p. 378

Pounds K.A., et al., 1993, MNRAS 260, 77

Radick R.R., Lockwood G.W., Thompson D.T., et al., 1983, PASP 95, 621

Radick R.R., Thompson D.T., Lockwood G.W., Duncan D.K., Baggett W.E., 1987, ApJ 321, 459

Rucinski S.M., 1987, PASP 99, 487

Rutten R.G.M., Schrijver C.J., Lemmens A.F.P., Zwaan C., 1991, A\&A 252, 203

Saar S.H., 1990, in: Solar Photosphere: Structure, Convection and Magnetic Fields. In: Stenflo J.D. (ed.). Kluwer, Dordrecht, p. 427

Simon T., Drake S.A., 1989, ApJ 346, 303

Soderblom D.K., 1985, AJ 90, 2103

Soderblom D.K., Stauffer J.R., MacGregor K.B., Jones B.F., 1993, ApJ 409, 624

Stępień K., 1989, A\&A 210, 273

Stępień K., 1991, in: The Sun and Cool Stars: activity, magnetism, dynamos. In: Tuominen I., Moss D., Rüdiger G. (eds.). Springer, Berlin, p. 280

Stẹpień K., 1994, A\&A 292, 191

Vogt N., Geisse H.S., Rojas S., 1981, A\&AS 46, 7

Wilson O.C., 1966, ApJ 144, 695

Zhang Q., Soon W.H., Baliunas S.L., Lockwood G.W., Skiff B.A., Radick R.R., 1994, ApJ 427, L111

Ziegler K., Shirley M., Francam B., Florence W., Hall D.S., 1984, IBVS, No. 2619 